\title{
Harmonic mappings and distance function
}

\author{
DAVID KALAJ
}

\begin{abstract}
We prove the following theorem: every quasiconformal harmonic mapping between two plane domains with $C^{1, \alpha}(\alpha<1)$ and, respectively, $C^{1,1}$ compact boundary is bi-Lipschitz. This theorem extends a similar result of the author [10] for Jordan domains, where stronger boundary conditions for the image domain were needed. The proof uses distance function from the boundary of the image domain.
\end{abstract}

Mathematics Subject Classification (2010): 58E20 (primary); 30C62 (secondary).

\section{Introduction and statement of the main result}

We say that a function $u: D \rightarrow \mathbb{R}$ is ACL (absolutely continuous on lines) in the region $D \subset \mathbb{R}^{2}$, if for every closed rectangle $R \subset D$ with sides parallel to the $x$ and $y$-axes, $u$ is absolutely continuous on a.e. horizontal and a.e. vertical line in $R$. Such a function has, of course, partial derivatives $u_{x}$ and $u_{y}$ a.e. in $D$. A homeomorphism $f: D \rightarrow G$, where $D$ and $G$ are subdomains of the complex plane $\mathbb{C}$, is said to be $K$-quasiconformal ( $K$-q.c), for $K \geq 1$, if $f$ is ACL and

$$
|\nabla f(z)| \leq K l(\nabla f(z)) \quad \text { a.e. on } D
$$

where

$$
|\nabla f(x)|:=\max _{|h|=1}|\nabla f(x) h|=\left|f_{z}\right|+\left|f_{\bar{z}}\right|
$$

and

$$
l(\nabla f(z)):=\min _{|h|=1}|\nabla f(z) h|=\left|f_{z}\right|-\left|f_{\bar{z}}\right|
$$

(cf. [1, pages 23-24] and [22]). Note that, condition (1.1) can be written as

$$
\left|f_{\bar{z}}\right| \leq k\left|f_{z}\right| \quad \text { a.e. on } D \text {, where } k=\frac{K-1}{K+1} \text { i.e. } K=\frac{1+k}{1-k}
$$

Received October 9, 2009; accepted in revised form May 26, 2010. 
or in its equivalent form

$$
|\nabla f(z)|^{2} \leq K J_{f}(z), z \in \mathbb{U},
$$

where $J_{f}$ is the Jacobian of $f$.

A function $w$ is called harmonic in a region $D$ if it has form $w=u+i v$ where $u$ and $v$ are real-valued harmonic functions on $D$. If $D$ is simply connected, then there are two analytic functions $g$ and $h$ defined on $D$ such that $w$ has the representation

$$
w=g+\bar{h} .
$$

If $w$ is a harmonic univalent function then, by Lewy's theorem (see [23]), $w$ has a non-vanishing Jacobian and consequently, according to the inverse mapping theorem, $w$ is a diffeomorphism.

Let

$$
P(r, x)=\frac{1-r^{2}}{2 \pi\left(1-2 r \cos x+r^{2}\right)}
$$

denote the Poisson kernel. Then every bounded harmonic function $w$ defined on the unit disc $\mathbb{U}:=\{z:|z|<1\}$ has the representation

$$
w(z)=P[F](z)=\int_{0}^{2 \pi} P(r, x-\varphi) F\left(e^{i x}\right) d x,
$$

where $z=r e^{i \varphi}$ and $F$ is a bounded integrable function defined on the unit circle $S^{1}$.

In this paper we continue to study quasiconformal harmonic mappings. See [25] for the pioneering work on this topic, and [8] for related earlier results. In some recent papers, a lot of work have been done on this class of mappings ([3, 10-17, 19-21,24,26,28,29]). In these papers for the Lipschitz and the co-Lipschitz character is established quasiconformal harmonic mappings between plane domains with certain boundary conditions. In [32] the same problem is considered for hyperbolic harmonic quasiconformal selfmappings of the unit disk. Notice that, in general, quasi-symmetric self-mappings of the unit circle do not have a quasiconformal harmonic extension to the unit disk. In [25] an example is given of $C^{1}$ diffeomorphism of the unit circle onto itself whose Euclidean harmonic extension is not Lipschitz. Alessandrini and Nesi proved in [2] the following:

Proposition 1.1. Let $F: S^{1} \rightarrow \gamma \subset \mathbb{C}$ be an orientation-preserving diffeomorphism of class $C^{1}$ of $S^{1}$ onto a simple closed curve $\gamma$. Let $D$ be the bounded domain such that $\partial D=\gamma$. Let $w=P[F] \in C^{1}(\overline{\mathbb{U}} ; \mathbb{C})$. The mapping $w$ is a diffeomorphism of $U$ onto $D$ if and only if

$$
J_{w}>0 \text { everywhere on } S^{1} .
$$

From the inequalities (1.2) and (1.4), we easily deduce the following:

Corollary 1.2. Under the assumption of Proposition 1.1 the harmonic mapping $w$ is a diffeomorphism if and only if it is $K$-quasiconformal for some $K \geq 1$. 
In contrast to the case of the Euclidean metric, in the case of the hyperbolic metric, if $f: S^{1} \mapsto S^{1}$ is $C^{1}$ diffeomorphism, or more generally if $f: S^{n-1} \mapsto$ $S^{m-1}$ is a mapping with non-vanishing energy, then its hyperbolic harmonic extension is $C^{1}$ up to the boundary $([4,5])$.

To continue we need the definition of $C^{k, \alpha}$ Jordan curves $(k \in \mathbb{N}, 0<\alpha \leq 1)$. Let $\gamma$ be a rectifiable curve in the complex plane. Let $l$ be the length of $\gamma$. Let $g$ : $[0, l] \mapsto \gamma$ be an arc-length parametrization of $\gamma$. Then $|\dot{g}(s)|=1$ for all $s \in[0, l]$. We will say that $\gamma \in C^{k, \alpha}, k \in \mathbb{N}, 0<\alpha \leq 1$ if $g \in C^{k}$, and $M(k, \alpha):=$ $\sup _{t \neq s} \frac{\left|g^{(k)}(t)-g^{(k)}(s)\right|}{\mid t-s^{\alpha}}<\infty$. Notice this important fact: if $\gamma \in C^{1,1}$ then $\gamma$ has a curvature $\kappa_{z}$ for a.e. $z \in \gamma$ and ess $\sup \left\{\left|\kappa_{z}\right|: z \in \gamma\right\} \leq M(1,1)<\infty$.

This definition can be easily extended to an arbitrary $C^{k, \alpha}$ compact 1-dimensional manifold (not necessarily connected).

The starting point of this paper is the following proposition.

Proposition 1.3. Let $w=f(z)$ be a $K$-quasiconformal harmonic mapping between a Jordan domain $\Omega_{1}$ with $C^{1, \alpha}$ boundary and a Jordan domain $\Omega$ with $C^{1, \alpha}$ (respectively $C^{2, \alpha}$ ) boundary. Consider in addition $b \in \Omega_{1}$ and set $a=f(b)$. Then $w$ is Lipschitz (respectively co-Lipschitz). Moreover there exists a positive constant $c=c\left(K, \Omega, \Omega_{1}, a, b\right) \geq 1$ such that

$$
\left|f\left(z_{1}\right)-f\left(z_{2}\right)\right| \leq c\left|z_{1}-z_{2}\right|, \quad z_{1}, z_{2} \in \Omega_{1}
$$

and

$$
\frac{1}{c}\left|z_{1}-z_{2}\right| \leq\left|f\left(z_{1}\right)-f\left(z_{2}\right)\right|, \quad z_{1}, z_{2} \in \Omega_{1},
$$

respectively.

See [13] for the first part of Proposition 1.3 and [10] for its second part. In [10], it was conjectured that the second part of Proposition 1.3 remains true if we assume that $\Omega$ has $C^{1, \alpha}$ boundary only. Notice that the proof of Proposition 1.3 relies on the Kellogg-Warschawski theorem $([6,33,34])$ from the theory of conformal mappings, which asserts that if $w$ is a conformal mapping of the unit disk onto a domain $\Omega \in C^{k, \alpha}$, then $w^{(k)}$ has a continuous extension to the boundary $(k \in \mathbb{N})$. It also depended on Mori's theorem from the theory of quasiconformal mappings, which deals with the Hölder character of quasiconformal mappings between plane domains (see [1,31]). In addition, Lemma 3.2 below is needed.

Using a different approach, we will extend here as stated in Theorem 1.4 the second part of Proposition 1.3 to the case of image domains with $C^{1,1}$ boundary. The proof of Theorem 1.4, given in the last section, is different form the proof of second part of Proposition 1.3, and the use of the Kellogg-Warschawski theorem for the second derivative ([34]) is avoided. The distance function is used and hence a "weaker" smoothness of the boundary of image domain is needed.

Theorem 1.4 (The main theorem). Let $w=f(z)$ be a $K$-quasiconformal harmonic mapping from the unit disk $\mathbb{U}$ to a Jordan domain $\Omega$ with $C^{1,1}$ boundary. Set 
$a=f(0)$. Then $w$ is co-Lipschitz. More precisely, there exists a positive constant $c=c(K, \Omega, a) \geq 1$ such that

$$
\frac{1}{c}\left|z_{1}-z_{2}\right| \leq\left|f\left(z_{1}\right)-f\left(z_{2}\right)\right|, \quad z_{1}, z_{2} \in \Omega .
$$

Since the composition of a quasiconformal harmonic and a conformal mapping is itself quasiconformal harmonic, using Theorem 1.4 and Kellogg's theorem for the first derivative we obtain:

Corollary 1.5. Let $w=f(z)$ be a $K$-quasiconformal harmonic mapping between a plane domain $\Omega_{1}$ with $C^{1, \alpha}$ compact boundary and a plane domain $\Omega$ with $C^{1,1}$ compact boundary. Consider $a_{0} \in \Omega_{1}$ and set $b_{0}=f\left(a_{0}\right)$. Then $w$ is bi-Lipschitz. Moreover there exists a positive constant $c=c\left(K, \Omega, \Omega_{1}, a_{0}, b_{0}\right) \geq 1$ such that

$$
\frac{1}{c}\left|z_{1}-z_{2}\right| \leq\left|f\left(z_{1}\right)-f\left(z_{2}\right)\right| \leq c\left|z_{1}-z_{2}\right|, \quad z_{1}, z_{2} \in \Omega_{1} .
$$

Proof of Corollary 1.5. Let $b=f(a) \in \partial \Omega$. Since $\partial \Omega \in C^{1,1}$, it follows that there exists a $C^{1,1}$ Jordan curve $\gamma_{b} \subset \bar{\Omega}$, whose interior $D_{b}$ lies in $\Omega$, and $\partial \Omega \cap \gamma_{b}$ is a neighborhood of $b$. See [13, Theorem 2.1] for an explicit construction of such a Jordan curve. Let $D_{a}=f^{-1}\left(D_{b}\right)$, and take a conformal mapping $g_{a}$ of the unit disk onto $D_{a}$. Then $f_{a}=f \circ g_{a}$ is a quasiconformal harmonic mapping from the unit disk onto the $C^{1,1}$ domain $D_{b}$. From Theorem 1.4 it follows that $f_{a}$ is bi-Lipschitz, and from Kellogg's theorem it follows that $f=f_{a} \circ g_{a}^{-1}$ and its inverse $f^{-1}$ are Lipschitz in some small neighborhood of $a$ and of $b=f(a)$ respectively. This means that $\nabla f$ is bounded in some neighborhood of $a$. Since $\partial \Omega_{1}$ is a compact, we deduce that $\nabla f$ is bounded in $\partial \Omega_{1}$. The same holds for $\nabla f^{-1}$ with respect to $\partial \Omega$. This implies that $f$ is bi-Lipschitz.

ACKNOWLEDGEMENTS. I thank the referee for providing constructive comments and help in improving the contents of this paper.

\section{Auxiliary results}

Let $\Omega$ be a domain in $\mathbb{R}^{2}$ having non-empty boundary $\partial \Omega$. The distance function from the boundaryis defined by

$$
d(x)=\operatorname{dist}(x, \partial \Omega) .
$$

Let $\Omega$ be bounded and assume $\partial \Omega \in C^{1,1}$. These conditions on $\Omega$ imply that $\partial \Omega$ satisfies the following: at a.e. point $z \in \partial \Omega$ there exists a disk $D=D\left(w_{z}, r_{z}\right)$ depending on $z$ such that $\bar{D} \cap(\mathbb{C} \backslash \Omega)=\{z\}$. Moreover $\mu:=\operatorname{ess} \inf \left\{r_{z}, z \in\right.$ 
$\partial \Omega\}>0$. It is easy to show that $\mu^{-1}$ bounds the curvature of $\partial \Omega$, which means that $\frac{1}{\mu} \geq \kappa_{z}$, for $z \in \partial \Omega$. Here $\kappa_{z}$ denotes the curvature of $\partial \Omega$ at $z \in \partial \Omega$. Under the above conditions, we have $d \in C^{1,1}\left(\Gamma_{\mu}\right)$, where $\Gamma_{\mu}=\{z \in \bar{\Omega}: d(z)<\mu\}$ and for $z \in \Gamma_{\mu}$ there exists $\omega(z) \in \partial \Omega$ such that

$$
\nabla d(z)=\boldsymbol{v}_{\omega(z)},
$$

where $\boldsymbol{v}_{\omega(z)}$ denotes the inner normal vector to the boundary $\partial \Omega$ at the point $\omega(z)$. See [7, Section 14.6] for the details.

Lemma 2.1. Let $w: \Omega_{1} \mapsto \Omega$ be a $K$-quasiconformal mapping and set $\chi=$ $-d(w(z))$. Then

$$
|\nabla \chi| \leq|\nabla w| \leq K|\nabla \chi|
$$

in $w^{-1}\left(\Gamma_{\mu}\right)$ for $\mu>0$ such that $1 / \mu>\kappa_{0}=\operatorname{ess} \sup \left\{\left|\kappa_{z}\right|: z \in \partial \Omega\right\}$.

Proof. Observe first that $\nabla d$ is a unit vector. From the identity $\nabla \chi=-\nabla d \cdot \nabla w$ it follows that

$$
|\nabla \chi| \leq|\nabla d||\nabla w|=|\nabla w|
$$

For a non-singular matrix $A$ we have

$$
\begin{aligned}
\inf _{|x|=1}|A x|^{2} & =\inf _{|x|=1}\langle A x, A x\rangle=\inf _{|x|=1}\left\langle A^{T} A x, x\right\rangle \\
& =\inf \left\{\lambda: \exists x \neq 0, A^{T} A x=\lambda x\right\} \\
& =\inf \left\{\lambda: \exists x \neq 0, A A^{T} A x=\lambda A x\right\} \\
& =\inf \left\{\lambda: \exists y \neq 0, A A^{T} y=\lambda y\right\}=\inf _{|x|=1}\left|A^{T} x\right|^{2} .
\end{aligned}
$$

We next denote that $(\nabla \chi)^{T}=-(\nabla w)^{T} \cdot(\nabla d)^{T}$, therefore for $x \in w^{-1}\left(\Gamma_{\mu}\right)$ we obtain

$$
|\nabla \chi| \geq \inf _{|e|=1}\left|(\nabla w)^{T} e\right|=\inf _{|e|=1}|\nabla w e|=l(w) \geq K^{-1}|\nabla w| .
$$

The proof of (2.3) is complete.

Lemma 2.2. Let $\left\{e_{1}, e_{2}\right\}$ be the canonical basis of the space $\mathbb{R}^{2}$. Let $w: \Omega_{1} \mapsto \Omega$ be a twice differentiable mapping and let $\chi=-d(w(z))$. Then

$$
\Delta \chi\left(z_{0}\right)=\frac{\kappa_{w_{0}}}{1-\kappa_{w_{0}} d\left(w\left(z_{0}\right)\right)}\left|\left(O_{z_{0}} \nabla w\left(z_{0}\right)\right)^{T} e_{1}\right|^{2}-\left\langle(\nabla d)\left(w\left(z_{0}\right)\right), \Delta w\right\rangle,
$$

where $z_{0} \in w^{-1}\left(\Gamma_{\mu}\right), \omega_{0} \in \partial \Omega$ with $\left|w\left(z_{0}\right)-\omega_{0}\right|=\operatorname{dist}\left(w\left(z_{0}\right), \partial \Omega\right), \mu>0$ such that $1 / \mu>\kappa_{0}=\operatorname{ess} \sup \left\{\left|\kappa_{z}\right|: z \in \partial \Omega\right\}$ and $O_{z_{0}}$ is an orthogonal transformation. 
Proof. Let $v_{\omega_{0}}$ be the inner unit normal vector to $\gamma$ at the point $\omega_{0} \in \gamma$. Let $O_{z_{0}}$ be an orthogonal transformation that takes the vector $e_{2}$ to $v_{\omega_{0}}$. In complex notations one has:

$$
O_{z_{0}} w=-i v_{\omega_{0}} w
$$

Take $\tilde{\Omega}:=O_{z_{0}} \Omega$. Let $\tilde{d}$ be the distance function for $\tilde{\Omega}$. Then

$$
d(w)=\tilde{d}\left(O_{z_{0}} w\right)=\operatorname{dist}\left(O_{z_{0}} w, \partial \tilde{\Omega}\right) .
$$

Therefore $\chi(z)=-\tilde{d}\left(O_{z_{0}}(w(z))\right)$. Furthermore

$$
\begin{aligned}
\Delta \chi(z)= & -\sum_{i=1}^{2}\left(D^{2} \tilde{d}\right)\left(O_{z_{0}}(w(z))\right)\left(O_{z_{0}} \nabla w(z) e_{i}, O_{z_{0}} \nabla w(z) e_{i}\right) \\
& -\langle\nabla d(w(z)), \Delta w(z)\rangle .
\end{aligned}
$$

To continue, we make use of the following proposition.

Proposition 2.3 ([7, Lemma 14.17]). Let $\Omega$ be bounded and assume $\partial \Omega \in C^{1,1}$. Then, with notation as in Lemma 2.2, we have

$$
\left(D^{2} \tilde{d}\right)\left(O_{z_{0}} w\left(z_{0}\right)\right)=\operatorname{diag}\left(\frac{-\kappa_{\omega_{0}}}{1-\kappa_{\omega_{0}} d}, 0\right)=\left(\begin{array}{cc}
\frac{-\kappa_{\omega_{0}}}{1-\kappa_{\omega_{0}} d} & 0 \\
0 & 0
\end{array}\right),
$$

where $\kappa_{\omega_{0}}$ denotes the curvature of $\partial \Omega$ at $\omega_{0} \in \partial \Omega$.

Applying (2.7) we have

$$
\begin{aligned}
& \sum_{i=1}^{2}\left(D^{2} \tilde{d}\right)\left(O_{z_{0}}\left(w\left(z_{0}\right)\right)\right)\left(O_{z_{0}}\left(\nabla w\left(z_{0}\right)\right) e_{i}, O_{z_{0}}\left(\nabla w\left(z_{0}\right)\right) e_{i}\right) \\
& =\sum_{i=1}^{2} \sum_{j, k=1}^{2} D_{j, k} \tilde{d}\left(O_{z_{0}}\left(w\left(z_{0}\right)\right)\right) D_{i}\left(O_{z_{0}} w\right)_{j}\left(z_{0}\right) \cdot D_{i}\left(O_{z_{0}} w\right)_{k}\left(z_{0}\right) \\
& =\sum_{j, k=1}^{2} D_{j, k} \tilde{d}\left(O_{z_{0}}\left(w\left(z_{0}\right)\right)\right)\left\langle\left(O_{z_{0}} \nabla w\left(z_{0}\right)\right)^{T} e_{j},\left(O_{z_{0}} \nabla w\left(z_{0}\right)\right)^{T} e_{k}\right\rangle \\
& =\frac{-\kappa_{\omega_{0}}}{1-\kappa_{\omega_{0}} \tilde{d}}\left|\left(O_{z_{0}} \nabla w\left(z_{0}\right)\right)^{T} e_{1}\right|^{2} .
\end{aligned}
$$

Finally we obtain

$$
\Delta \chi\left(z_{0}\right)=\frac{\kappa_{\omega_{0}}}{1-\kappa_{\omega_{0}} \tilde{d}}\left|\left(O_{z_{0}} \nabla w\left(z_{0}\right)\right)^{T} e_{1}\right|^{2}-\left\langle(\nabla d)\left(w\left(z_{0}\right)\right), \Delta w\right\rangle .
$$




\section{Proof of the main theorem}

The main step to establish the main theorem is the following lemma.

Lemma 3.1. Let $w=f(z)$ be a $K$-quasiconformal mapping of the unit disk onto $a C^{1,1}$ Jordan domain $\Omega$ satisfying the differential inequality

$$
|\Delta w| \leq B|\nabla w|^{2}, \quad B \geq 0
$$

for some $B \geq 0$. Assume in addition that $w(0)=a_{0} \in \Omega$. Then there exists $a$ constant $C(K, \Omega, B, a)>0$ such that

$$
\left|\frac{\partial w}{\partial r}(t)\right| \geq C\left(K, \Omega, B, a_{0}\right) \text { for almost every } t \in S^{1} .
$$

Proof. Let us find $A>0$ such that the function $\varphi_{w}(z)=-\frac{1}{A}+\frac{1}{A} e^{-\operatorname{Ad}(w(z))}$ is subharmonic on $\left\{z: d(w(z))<\frac{1}{2 \kappa_{0}}\right\}$, where

$$
\kappa_{0}=\operatorname{ess} \sup \left\{\left|\kappa_{w}\right|: w \in \gamma\right\} .
$$

Let $\chi=-d(w(z))$. Combining (2.3), (2.5) and (3.1) we get

$$
|\Delta \chi| \leq 2 \kappa_{0}|\nabla w|^{2}+B|\nabla w|^{2} \leq\left(2 \kappa_{0}+B\right) K^{2}|\nabla \chi|^{2} .
$$

Take

$$
g(t)=-\frac{1}{A}+\frac{1}{A} e^{A t} .
$$

Then $\varphi_{w}(z)=g(\chi(z))$. Thus

$$
\Delta \varphi_{w}=g^{\prime \prime}(\chi)|\nabla \chi|^{2}+g^{\prime}(\chi) \Delta \chi .
$$

Since

$$
g^{\prime}(\chi)=e^{-\operatorname{Ad}(w(z))}
$$

and

$$
g^{\prime \prime}(\chi)=A e^{-A d(w(z))},
$$

it follows that

$$
\Delta \varphi_{w} \geq\left(A-\left(2 \kappa_{0}+B\right) K^{2}\right)|\nabla \chi|^{2} e^{-A d(u(z))} .
$$

In order to have $\Delta \varphi_{w} \geq 0$, it is enough to take

$$
A=\left(2 \kappa_{0}+B\right) K^{2} .
$$

Choosing

$$
\varrho=\max \left\{|z|: \operatorname{dist}(w(z), \gamma)=\frac{1}{2 \kappa_{0}}\right\},
$$

we have that $\varphi_{w}$ satisfies the conditions of the following generalization of the Hopf lemma ([9]): 
Lemma 3.2 ([10]). Let $\varphi$ satisfy $\Delta \varphi \geq 0$ in $R_{\varrho}=\{z: \varrho \leq|z|<1\}, 0<\varrho<1$, $\varphi$ be continuous on $\overline{R_{\varrho}}, \varphi<0$ in $R_{\varrho}, \varphi(t)=0$ for $t \in S^{1}$. Assume that the radial derivative $\frac{\partial \varphi}{\partial r}$ exists almost everywhere on $S^{1}$. Set $M(\varphi, \varrho)=\max _{|z|=\varrho} \varphi(z)$. Then the following inequality holds

$$
\frac{\partial \varphi(t)}{\partial r}>\frac{2 M(\varphi, \varrho)}{\varrho^{2}\left(1-e^{1 / \varrho^{2}-1}\right)} \text { for a.e. } t \in S^{1} .
$$

We will make use of (3.9), but under some improvement for the class of quasiconformal harmonic mappings. The idea is to make the right-hand side of (3.9) independent of the mapping $w$ for $\varphi=\varphi_{w}$.

We will say that a quasiconformal mapping $f: \mathbb{U} \mapsto \Omega$ is normalized if $f(1)=w_{0}, f\left(e^{2 \pi i / 3}\right)=w_{1}$ and $f\left(e^{-2 \pi i / 3}\right)=w_{2}$, where $w_{0} w_{1}, w_{1} w_{2}$ and $w_{2} w_{0}$ are arcs of $\gamma=\partial \Omega$ having the same length $|\gamma| / 3$.

In what follows we will prove that, for the class $\mathcal{H}(\Omega, K, B)$ of normalized $K$-quasiconformal mappings, satisfying (3.1) for some $B \geq 0$, and mapping the unit disk onto the domain $\Omega$, the inequality (3.9) holds uniformly (see (3.10)).

Let

$$
\varrho:=\sup \left\{|z|: \operatorname{dist}(w(z), \gamma)=\frac{1}{2 \kappa_{0}}, w \in \mathcal{H}(\Omega, K, B)\right\} .
$$

Then there exists a sequence $\left\{w_{n}\right\}, w_{n} \in \mathcal{H}(\Omega, K, B)$ such that

$$
\varrho_{n}=\max \left\{|z|: \operatorname{dist}\left(w_{n}(z), \gamma\right)=\frac{1}{2 \kappa_{0}}\right\},
$$

and

$$
\varrho=\lim _{n \rightarrow \infty} \varrho_{n} .
$$

Now notice that if $w_{n}$ is a sequence of normalized $K$-quasiconformal mappings of the unit disk onto $\Omega$ then, up to taking a subsequence, $w_{n}$ is a locally uniformly convergent sequence converging to some quasiconformal mapping $w \in$ $\mathcal{H}(\Omega, K, B)$. Under the condition on the boundary of $\Omega$, by [27, Theorem 4.4] this sequence is uniformly convergent on $\mathbb{U}$. Then there exists a sequence $z_{n}$ such that $\operatorname{dist}\left(w_{n}\left(z_{n}\right), \gamma\right)=\frac{1}{2 \kappa_{0}}, \lim _{n \rightarrow \infty} z_{n}=z_{0}$ and $\varrho=\left|z_{0}\right|$. Since $w_{n}$ converges uniformly to $w$, it follows that $\lim _{n \rightarrow \infty} w_{n}\left(z_{n}\right)=w\left(z_{0}\right)$, and $\operatorname{dist}\left(w\left(z_{0}\right), \gamma\right)=\frac{1}{2 \kappa_{0}}$. This implies that $\varrho<1$. Let now

$$
M(\varrho):=\sup \left\{M\left(\varphi_{w}, \varrho\right), w \in \mathcal{H}(\Omega, K, B)\right\} .
$$

Using a similar argument we obtain that there exists a uniformly convergent sequence $w_{n}$, converging to a mapping $w_{0}$, such that

$$
M(\varrho)=\lim _{n \rightarrow \infty} M\left(\varphi_{w_{n}}, \varrho\right)=M\left(\varphi_{w_{0}}, \varrho\right) .
$$


Thus

$$
M(\varrho)<0 .
$$

Placing $M(\varrho)$ instead of $M(\varrho, \varphi)$ and $\varphi_{w}$ instead of $\varphi$ in (3.9), we obtain

$$
\frac{\partial \varphi_{w}(t)}{\partial r}>\frac{2 M(\varrho)}{\varrho^{2}\left(1-e^{1 / \varrho^{2}-1}\right)}:=C(K, \Omega, B) \text { for a.e. } t \in S^{1} .
$$

To continue observe that

$$
\frac{\partial \varphi_{w}(t)}{\partial r}=e^{A d(w(z))}|\nabla d|\left|\frac{\partial w}{\partial r}(t)\right|=e^{A d(w(z))}\left|\frac{\partial w}{\partial r}(t)\right| .
$$

Combining (3.8) and (3.10) we obtain for a.e. $t \in S^{1}$

$$
\left|\frac{\partial w}{\partial r}(t)\right|=e^{-A d(w(z))} \frac{\partial \varphi_{w}(t)}{\partial r} \geq e^{-K^{2}} \frac{2 M(\varrho)}{\varrho^{2}\left(1-e^{1 / \varrho^{2}-1}\right)} .
$$

Lemma 3.1 is now proved for a normalized mapping $w$. If $w$ is not normalized then we take the composition of $w$ and an approprieate Möbius transformation in order to obtain the desired inequality. The proof of Lemma 3.1 is complete.

Conclusion of the proof of Theorem 1.4. In this setting $w$ is harmonic, therefore $B=0$. Assume first that $w \in C^{1}(\overline{\mathbb{U}})$. Let $l(\nabla w)(t)=|| w_{z}(t)|-| w_{\bar{z}}(t)||$. Since $w$ is $K$-quasiconformal, according to (3.2) we have

$$
l(\nabla w)(t) \geq \frac{|\nabla w(t)|}{K} \geq \frac{\left|\frac{\partial w}{\partial r}(t)\right|}{K} \geq \frac{C\left(K, \Omega, 0, a_{0}\right)}{K}
$$

for $t \in S^{1}$. Therefore, having in mind Lewy's theorem ([23]), which states that $\left|w_{z}\right|>\left|w_{\bar{z}}\right|$ for $z \in \mathbb{U}$, we obtain for $t \in S^{1}$ that $\left|w_{z}(t)\right| \neq 0$ and hence

$$
\frac{1}{\left|w_{z}\right|} \frac{C\left(K, \Omega, 0, a_{0}\right)}{K}+\frac{\left|w_{\bar{z}}\right|}{\left|w_{z}\right|} \leq 1, t \in S^{1} .
$$

Since $w \in C^{1}(\overline{\mathbb{U}})$, it follows that the functions

$$
a(z):=\frac{\overline{w_{\bar{z}}}}{w_{z}}, \quad b(z):=\frac{1}{w_{z}} \frac{C\left(K, \Omega, 0, a_{0}\right)}{K}
$$

are well-defined holomorphic functions in the unit disk having a continuous extension to the boundary. As $|a|+|b|$ is bounded on the unit circle by 1 , it follows that it is bounded on the whole unit disk by 1 because

$$
|a(z)|+|b(z)| \leq P\left[|a|_{S^{1}}\right](z)+P\left[|b|_{S^{1}}\right](z)=P\left[|a|_{S^{1}}+|b|_{S^{1}}\right](z), \quad z \in \mathbb{U} .
$$


This in turn implies that for every $z \in \mathbb{U}$

$$
l(\nabla w)(z) \geq \frac{C\left(K, \Omega, 0, a_{0}\right)}{K}=: C\left(\Omega, K, a_{0}\right) .
$$

This yields that

$$
C\left(K, \Omega, a_{0}\right) \leq \frac{\left|w\left(z_{1}\right)-w\left(z_{2}\right)\right|}{\left|z_{1}-z_{2}\right|}, \quad z_{1}, z_{2} \in \mathbb{U}
$$

Assume now that $w \notin C^{1}(\overline{\mathbb{U}})$. We begin with a definition.

Definition 3.3. Let $G$ be a domain in $\mathbb{C}$ and let $a \in \partial G$. We will say that $G_{a} \subset G$ is a d-neighborhood of $a$ if there exists a disk $D(a, r):=\{z:|z-a|<r\}$ such that $D(a, r) \cap G \subset G_{a}$.

Let $t=e^{i \beta} \in S^{1}$, so that $w(t) \in \partial \Omega$. Let $\gamma$ be an arc-length parametrization of $\partial \Omega$ with $\gamma(s)=w(t)$. Since $\partial \Omega \in C^{1,1}$, there exists a $\partial$-neighborhood $\Omega_{t}$ of $w(t)$ with $C^{1,1}$ Jordan boundary such that

$$
\Omega_{t}^{\tau}:=\Omega_{t}+i \gamma^{\prime}(s) \cdot \tau \subset \Omega \text {, and } \partial \Omega_{t}^{\tau} \subset \Omega \text { for } 0<\tau \leq \tau_{t}\left(\tau_{t}>0\right) .
$$

An example of a family $\Omega_{t}^{\tau}$ such that $\partial \Omega_{t}^{\tau} \in C^{1,1}$ and with the property (3.13) has been given in [13].

Let $a_{t} \in \Omega_{t}$ be arbitrary. Then $a_{t}+i \gamma^{\prime}(s) \cdot \tau \in \Omega_{t}^{\tau}$. Take $U_{\tau}=f^{-1}\left(\Omega_{t}^{\tau}\right)$. Let $\eta_{t}^{\tau}$ be a conformal mapping of the unit disk onto $U_{\tau}$ such that $\eta_{t}^{\tau}(0)=f^{-1}\left(a_{t}+\right.$ $\left.i \gamma^{\prime}(s) \cdot \tau\right)$, and $\arg \frac{d \eta_{t}^{\tau}}{d z}(0)=0$. Then the mapping

$$
f_{t}^{\tau}(z):=f\left(\eta_{t}^{\tau}(z)\right)-i \gamma^{\prime}(s) \cdot \tau
$$

is a harmonic $K$-quasiconformal mapping of the unit disk onto $\Omega_{t}$ satisfying the condition $f_{t}^{\tau}(0)=a_{t}$. Moreover

$$
f_{t}^{\tau} \in C^{1}(\overline{\mathbb{U}})
$$

Using the case $w \in C^{1}(\overline{\mathbb{U}})$, it follows that

$$
\left|\nabla f_{t}^{\tau}(z)\right| \geq C\left(K, \Omega_{t}, a_{t}\right) .
$$

On the other hand

$$
\lim _{\tau \rightarrow 0+} \nabla f_{t}^{\tau}(z)=\nabla\left(f \circ \eta_{t}\right)(z)
$$

on the compact sets of $\mathbb{U}$ as well as

$$
\lim _{\tau \rightarrow 0+} \frac{d \eta_{t}^{\tau}}{d z}(z)=\frac{d \eta_{t}}{d z}(z)
$$


where $\eta_{t}$ is a conformal mapping of the unit disk onto $U_{0}=f^{-1}\left(\Omega_{t}\right)$ with $\eta_{t}(0)=$ $f^{-1}\left(a_{t}\right)$. It follows that

$$
\left|\nabla f_{t}(z)\right| \geq C\left(K, \Omega_{t}, a_{t}\right)
$$

Applying the Schwarz reflexion principle to the mapping $\eta_{t}$ and using the formula

$$
\nabla\left(f \circ \eta_{t}\right)(z)=\nabla f \cdot \frac{d \eta_{t}}{d z}(z)
$$

it follows that in some $\partial$-neighborhood $\tilde{U}_{t}$ of $t \in S^{1}$ with smooth boundary where $\left(D\left(t, r_{t}\right) \cap \mathbb{U} \subset \tilde{U}_{t}\right.$ for some $\left.r_{t}>0\right)$, the function $f$ satisfies the inequality

$$
|\nabla f(z)| \geq \frac{C\left(K, \Omega_{t}, a_{t}\right)}{\max \left\{\left|\eta_{t}^{\prime}(\zeta)\right|: \zeta \in \overline{\tilde{U}_{t}}\right\}}=: \tilde{C}\left(K, \Omega_{t}, a_{t}\right)>0
$$

Since $S^{1}$ is a compact set, it can be covered by a finite family $\partial \tilde{U}_{t_{j}} \cap S^{1} \cap D\left(t, r_{t} / 2\right)$, $j=1, \ldots, m$. It follows that the inequality

$$
|\nabla f(z)| \geq \min \left\{\tilde{C}\left(K, \Omega_{t_{j}}, a_{t_{j}}\right): j=1, \ldots, m\right\}=: \tilde{C}\left(K, \Omega, a_{0}\right)>0
$$

holds in the annulus

$$
\tilde{R}=\left\{z: 1-\frac{\sqrt{3}}{2} \min _{1 \leq j \leq m} r_{t_{j}}<|z|<1\right\} \subset \bigcup_{j=1}^{m} \tilde{U}_{t_{j}} .
$$

This implies that the subharmonic function $S=|a(z)|+|b(z)|$ is bounded in $\mathbb{U}$. According to the maximum principle, it is bounded by 1 in the whole unit disk. This in turn implies again (3.12) and consequently

$$
\frac{C\left(K, \Omega, a_{0}\right)}{K}\left|z_{1}-z_{2}\right| \leq\left|w\left(z_{1}\right)-w\left(z_{2}\right)\right|, \quad z_{1}, z_{2} \in \mathbb{U} .
$$

\section{References}

[1] L. AhLFors, Lectures on Quasiconformal mappings, Van Nostrand Mathematical Studies, Vol. 10, D. Van Nostrand 1966.

[2] G. AlesSANDRINI and V. NeSI, Invertible harmonic mappings, beyond Kneser, Ann. Scuola Norm. Sup. Pisa Cl. Sci. (5) 8 (2009), 451-468.

[3] M. Arsenovic, V. Kojic, and M. Mateljevic, On lipschitz continuity of harmonic quasiregular maps on the unit ball in $\mathbb{R}^{n}$, Ann. Acad. Sci. Fenn. Math. 33 (2008), 315-318.

[4] P. LI, and L. TAM, Uniqueness and regularity of proper harmonic maps, Ann. of Math. (2) 137 (1993), 167-201.

[5] P. LI, and L. TAM, Uniqueness and regularity of proper harmonic maps. II, Indiana Univ. Math. J. 42 (1993), 591-635.

[6] G. M. Goluzin, Geometric function theory, Nauka Moskva, Russian, 1966. 
[7] D. Gilbarg and N. Trudinger, "Elliptic Partial Differential Equations of Second Order", Vol. 224, Second Edition, Springer 1977, 1983.

[8] W. HengarTneR and G. SCHOBER, Harmonic mappings with given dilatation, J. London Math. Soc. 33 (1986), 473-483.

[9] E. Hopf, A remark on linear elliptic differential equations of second order, Proc. Amer. Math. Soc. 3 (1952), 791-793.

[10] D. KalaJ, Lipschitz spaces and harmonic mappings, Ann. Acad. Sci. Fenn. Math. 34 (2009), 475-485.

[11] D. KALAJ, Quasiconformal harmonic functions between convex domains, Publ. Inst. Math. 76 (2004), 3-20.

[12] D. KALAJ, On harmonic quasiconformal self-mappings of the unit ball, Ann. Acad. Sci. Fenn. Math. 33 (2008), 1-11.

[13] D. KalaJ, Quasiconformal harmonic mapping between Jordan domains, Math. Z. 260 (2008), 237-252.

[14] D. KALAJ, On harmonic diffeomorphisms of the unit disc onto a convex domain, Complex Var. Theory Appl. 48 (2003), 175-187.

[15] D. KalaJ, On quasiregular mappings between smooth Jordan domains, J. Math. Anal. Appl. 362 (2010), 58-63.

[16] D. KALAJ and M. MATELJEVIĆ, Inner estimate and quasiconformal harmonic maps between smooth domains, J. Anal. Math. 100 (2006), 117-132.

[17] D. Kalaj and M. Mateljević, On certain nonlinear elliptic PDE and quasiconfomal maps between Euclidean surfaces, Potential Anal. 34 (2010), 13-22.

[18] D. Kalaj and M. MatelJević, On quasiconformal harmonic surfaces with rectifiable boundary, Complex Anal. Oper. Theory, to appear. doi: 10.1007/s11785-010-0062-9.

[19] D. KALAJ and M. PAVlović, Boundary correspondence under harmonic quasiconformal homeomorfisms of a half-plane, Ann. Acad. Sci. Fenn. Math. 30 (2005), 159-165.

[20] D. KALAJ and M. PAVlović, On quasiconformal self-mappings of the unit disk satisfying the Poisson equation, Trans. Amer. Math. Soc. 363 (2011), 4043-4061.

[21] M. KNeZEVIC and M. MATELJEVIC, On the quasi-isometries of harmonic quasiconformal mappings, J. Math. Anal. Appl. 334 (2007), 404-413.

[22] O. Lehto and K. I. Virtanen, "Quasiconformal Mapping", Springer-Verlag, Berlin and New York, 1973.

[23] H. LEWY, On the non-vanishing of the Jacobian in certain in one-to-one mappings, Bull. Amer. Math. Soc. 42 (1936), 689-692.

[24] V. MANOJLOVIĆ, Bi-lipshicity of quasiconformal harmonic mappings in the plane, Filomat 23 (2009), 85-89.

[25] O. MARTIO, On harmonic quasiconformal mappings, Ann. Acad. Sci. Fenn. Ser. A I 425 (1968), 3-10.

[26] M. MateljeVic and M. Vuorinen, On harmonic quasiconformal quasi-isometries, J. Inequalities Appl. 2010 (2010), Article ID 178732, 19 p.

[27] R. NÄKKI and B. PALKA, Boundary regularity and the uniform convergence of quasiconformal mappings, Comment. Math. Helv. 54 (1979), 458-476.

[28] Partyka D.; Sakan, K. On bi-Lipschitz type inequalities for quasiconformal harmonic mappings, Ann. Acad. Sci. Fenn. Math. 32 (2007), 579-594.

[29] M. PAVLOVIĆ, Boundary correspondence under harmonic quasiconformal homeomorfisms of the unit disc, Ann. Acad. Sci. Fenn. Math. 27 (2002), 365-372.

[30] C. Pommerenke, "Boundary Behaviour of Conformal Maps", Springer-Verlag, New York, 1992.

[31] C. WANG, A sharp form of Mori's theorem on Q-mappings, Kexue Jilu 4 (1960), 334-337.

[32] T. WAN, Constant mean curvature surface, harmonic maps, and universal Teichmüller space, J. Differential Geom. 35 (1992), 643-657.

[33] S. E. WARSCHAWSKI, On differentiability at the boundary in conformal mapping, Proc. Amer. Math. Soc. 12 (1961), 614-620. 
[34] S. E. WARSCHAWSKI, On the higher derivatives at the boundary in conformal mapping, Trans. Amer. Math. Soc. 38 (1935), 310-340.

\author{
University of Montenegro \\ Faculty of Natural Sciences \\ and Mathematics \\ Cetinjski put b.b. \\ 81000 Podgorica, Montenegro \\ davidk@t-com.me
}

